\title{
Un phénomène de concentration évanescente pour des flots non-stationnaires incompressibles en dimension deux
}

\author{
S. Alinhac \\ Université de Paris-Sud, Département de Mathématiques, F-91405 Orsay Cedex, France
}

\begin{abstract}
We consider a sequence $v_{\varepsilon}$ of non-stationary solutions of the incompressible 2D-Euler equation, locally bounded in $L^{2}$. We prove that if the defect measure is supported in a one-dimensional set (in $\mathbb{R}^{3}$ ) of some special type (which we call "finite type"), the weak limit $v$ of $v_{\varepsilon}$ is a solution of the Euler equations: our theorem is of the type "concentration-cancellation".
\end{abstract}

\section{Introduction}

Dans l'étude du problème de Cauchy pour les équations d'Euler incompressibles en dimension deux d'espace, Di Perna et Majda ont récemment introduit [1,2] le concept de «suite de solutions approchées» (qui implique notamment des estimations a priori précises), et, pour ces dernières, les notions de «mesure de défaut réduite» et d'«ensemble de concentration» [3] (voir les rappels de la Sect. 1 du présent travail).

Il s'agit, dans ces travaux, d'étudier précisément la façon dont la suite des vitesses $v_{\varepsilon}$ converge faiblement (mais non fortement) dans $L^{2}$ vers $v$. Un des phénomènes intéressant mis en évidence dans [3] est le phénomène de "concentration évanescente» (que les auteurs appellent «concentration-cancellation»): il arrive, si le défaut de convergence forte est «très petit» (en un sens à préciser), que la limite faible $v$ soit quand même solution des équations d'Euler (bien qu'on ne puisse pas passer à la limite banalement dans ce cas).

Cela correspond au fait que la mesure de défaut satisfait alors une certaine équation.

Dans le cas d'une suite de solutions stationnaires $v_{\varepsilon}(x)$, un théorème de ce type a été obtenu par Di Perna et Majda (Thèorème 3.1 de [3]), par une méthode de «troncatures-fantômes».

Nous présentons ici une condition suffisante, dans le cas général nonstationnaire. Comme Greengard et Thomann ont remarqué [5] que le Théorème 2 de [3] correspondait en fait à une situation de convergence forte de $v_{\varepsilon}$ vers $v$, il 
semble que ce résultat soit le premier de ce type dans le cas non-stationnaire. Il n'apparaît toutefois pas suffisant encore pour obtenir l'existence de solutions faibles du problème de l'évolution des nappes de tourbillon.

\section{Généralités et rappels}

1.1. Nous considérons l'équation d'Euler incompressible en dimension deux d'espace $\left(x=\left(x_{1}, x_{2}\right) \in \mathbb{R}^{2}\right)$

$$
\left\{\begin{array}{l}
\frac{\partial v}{\partial t}+\operatorname{div}(v \otimes v)=-\nabla p \\
\operatorname{div} v=0
\end{array}\right.
$$

où $v=\left(v^{1}, v^{2}\right)$ est la vitesse, $v \otimes v$ est la matrice $2 \times 2$ de terme général $v^{i} v^{j}, \operatorname{div}(v \otimes v)$ signifiant qu'on calcule la divergence de chaque ligne de la matrice.

Di Perna et Majda [2] ont introduit une notion de «suite de solutions approchées» $v_{\varepsilon}$, impliquant notamment des estimations (uniformes en $\varepsilon$ ) sur $v_{\varepsilon}$ et $\omega_{\varepsilon}=\partial_{1} v_{\varepsilon}^{2}-\partial_{2} v_{\varepsilon}^{1}$.

Nous considérons ici une suite $v_{\varepsilon}$ définie sur $\mathbb{R}^{2} \times[0, T]$, telle que, pour tout $R$, il existe $C_{R}$ avec

$$
\sup _{t \in[0, T]} \int_{|x| \leqq R}\left|v_{\varepsilon}(x, t)\right|^{2} d x \leqq C_{R} .
$$

Nous ne faisons aucune hypothèse sur $\omega_{\varepsilon}$, ni sur le comportement de $v$ à l'infini.

Quitte à extraire une sous-suite de la suite $v_{\varepsilon}$, on peut toujours supposer qu'il existe $v \in L_{\text {loc }}^{2}\left(\mathbb{R}^{2} \times[0, T]\right)$ et une matrice

$$
\mu=\left(\mu_{i j}\right) \text { de mesure de Radon }
$$

telles que

$$
v_{\varepsilon} \rightarrow v \text { faiblement dans } L^{2}
$$

$$
v_{\varepsilon}^{i} v_{\varepsilon}^{j} \rightarrow \mu_{i j} \quad \text { au sens de la convergence vague des mesures. }
$$

Depuis le travail de Lions [6], il est coutumier d'introduire aussi la messure $\sigma$ définie par:

$$
\varphi \in C_{\text {comp }}^{0}, \quad \int \varphi(x)\left|\left(v_{\varepsilon}-v\right)(x)\right|^{2} d x \rightarrow\langle\varphi, \sigma\rangle,
$$

qui est appelée «mesure de défaut» (cf. aussi [4]). Bien entendu, $\sigma$ est positive et en fait $\sigma=\mu_{11}-\left(v^{1}\right)^{2}+\mu_{22}-\left(v^{2}\right)^{2}$.

Rappelons enfin quelques définitions de Di Perna et Majda [2,3]: pour tout borélien $E$, on pose:

$$
\theta(E)=\lim _{\varepsilon \rightarrow 0} \sup \int_{E}\left|v_{\varepsilon}-v\right|^{2} .
$$

La fonction $\theta$ est dite «mesure de défaut réduite», bien que ce ne soit pas une mesure.

$\operatorname{Si} \theta(E)=0$, on dit que $E$ est un «ensemble de convergence» (sous-entendu: forte dans $L^{2}$, de $v_{\varepsilon}$ vers $v$ ).

D'autre part, $E$ est un «ensemble de concentration» pour $\left\{v_{\varepsilon}\right\}$ si son complémentaire $E^{c}$ est réunion d'une suite croissante $F_{1} \subset F_{2} \subset \ldots$ de fermés de convergence. 
1.2. Nous souhaitons répondre à la question: si une suite $\left\{v_{\varepsilon}\right\}$ satisfaisant (1.1.2), (1.1.3) est formée de solutions approchées de (1.1.1), la limite faible $v$ est-elle une solution de (1.1.1)?

On trouve dans la littérautre deux types de réponses à cette question:

a. Des hypothèses supplémentaires de régularité sur $v_{\varepsilon}$ peuvent impliquer qu'en fait $v_{\varepsilon} \rightarrow v$ fortement dans $L^{2}$ (auquel cas on peut "passer à la limite» dans l'équation), ou qu'au moins $\left\{v_{\varepsilon}\right\}$ est concentrée sur de très petits ensembles.

Les résultats de Greengard et Thomann [5] et les Théorèmes 1 et 3 de Di Perna et Majda [3] sont de ce type.

b. Dans certains cas, il est possible de montrer que $v$ est solution de (1.1.1), grâce à une information du type obtenu en a: c'est la démarche du Théorème 3.1 de [3], correspondant au phénomène de "concentration évanescente».

Il apparaît en b la difficulté suivante: il ne semble pas possible d'obtenir des énoncés portant sur la seule dimension (de Hausdorff) d'un ensemble de concentration. Il faut en réalité faire une hypothèse sur la géométrie d'une famille d'ensembles de convergence qui épuise $E^{c}$. En effet, si $E$ est un ensemble de concentration et $F$ un fermé de mesure (de Lebesgue dans $\mathbb{R}^{3}$ ) nulle, $E \backslash F$ est encore un ensemble de concentration; dans le cas stationnaire considéré au Théorème 3.1 de [3], si par exemple $\sigma$ est la mesure de Dirac portée par une droite $F$ du plan, on voit tout de suite que $F$ est un ensemble de concentration, mais on peut aussi bien dire que $\phi$ en est un, auquel cas le théorème devrait s'appliquer, ce qui n'est pas.

Au paragraphe 3 , nous présentons un théorème de type $b$, qui semble être le premier résultat de ce type dans le cas non-stationnaire. Auparavant, nous allons définir la notion d'«ensemble de type fini» utilisée dans le théorème.

\section{Ensemble de type fini du plan par rapport à une direction}

Soit $E$ un ensemble relativement compact du plan $\mathbb{R}^{2}$, où les coordonnées sont notées $(x, t)$. Dans ce qui suit, la direction de l'axe des temps joue un rôle particulier.

\subsection{Quelques définitions}

Définition 2.1.1. On dit que $E$ est de type 0 s'il existe un nombre fini de compacts $K_{1}, \ldots, K_{N}, K_{i} \subset \mathbb{R}, K_{i}$ de mesure nulle, et des fonctions $\psi_{1}(x, t), \ldots, \psi_{N}(x, t)$ de la forme $\psi_{i} \equiv t$ ou $\psi_{i}(x, t)=x-\varphi_{i}(t)$ (pour une fonction $\varphi_{i}$ lipschitzienne à support compact sur $\mathbb{R}$ ) tels que

$$
\begin{aligned}
& \text { pour tous ouvert } \omega_{1}, \ldots, \omega_{N} \text { de } \mathbb{R}, \omega_{i} \supset K_{i}, \\
& \qquad E \subset \bigcup_{i} \psi_{i}^{-1}\left(\omega_{i}\right) .
\end{aligned}
$$
dessus,

On dit que $E$ est de type $k(k \in \mathbb{N}, k \geqq 1)$ si, avec les mêmes notations que ci-

$$
E \backslash \bigcup_{i} \psi_{i}^{-1}\left(\omega_{i}\right) \quad \text { est de type } k-1 \text {. }
$$

Bien entendu, si $F \subset E$ et $E$ est de type $k, F$ est lui-même de type $k$.

Définition 2.1.2. $E$ est de type fini s'il est de type $k$ pour un certain $k$. 


\subsection{Exemples d'ensembles de type $k$}

\subsubsection{Courbes $C^{1}$.}

Lemme. Soit $\{x(s), t(s)\}(s \in[0,1])$ un segment de courbe de classe $C^{1}$. Alors l'image $\Gamma$ est de type 1.

Preuve. Soit $C=\left\{s, t^{\prime}(s)=0\right\}: C$ est un compact, et son image $K=t(C)$ est un compact de mesure nulle, d'après le Théorème de Sarde.

Soit $\omega$ un ouvert, $\omega \supset K$ : l'ensemble $\widetilde{C}=[0,1] \backslash t^{-1}(\omega)$ est un compact. Pour tout $s_{0} \in \tilde{C}$, il existe un voisinage $V$ de $s_{0}$, un voisinage $T$ de $t_{0}=t\left(s_{0}\right)$ et une fonction $C^{1} s=s(t)$ sur $T$ tels que si $t=t(s)$ pour $s \in V$, alors $s=s(t)$ (d'après le théorème des fonctions implicites). En extrayant un recouvrement de $\widetilde{C} \operatorname{par} \operatorname{des} V_{j}$ [avec des $T_{j}$, $s_{j}(t)$ associés], on voit que tout point $(x(s), t(s))$ de $\Gamma$ pour lequel $t \notin \omega$ est contenu dans la réunion des arcs $x=x\left(s_{j}(t)\right)$.

Remarquons qu'il ne semble pas possible d'étendre le lemme au cas de courbes Lipschitziennes.

2.2.2. Spirales. La spirale $S$ d'équation $\varrho=e^{-\theta}(\theta \geqq 0)$ en «coordonnées polaires» $(\varrho, \theta)$ est de type 1 : en effet, la suite $K$ des valeurs critiques de $t$ converge vers 0 , et pour tout $\omega \supset K, S \backslash t^{-1}(\omega)$ est formée d'un nombre fini (dépendant de $\omega$ !) de graphes réguliers.

\subsubsection{Bouquets de droites. Soit $\left\{\lambda_{n}\right\}$ une suite, $\lambda_{n} \rightarrow 0$.}

L'ensemble $E=\left\{(x, t), \exists n, x=\lambda_{n} t\right\}$ est (localement) de type 1; en effet, pour tout $\omega \supset 0, E \backslash x^{-1}(\omega)$ est constitué d'un nombre fini de droites.

On peut créer des ensembles de type 2 par les mêmes procédés, en «accumulant» convenablement des ensembles de type 1 , etc....

Par ces quelques exemples, on a simplement voulu montrer que la condition «de type fini», qui exclut, parmi les ensembles du plan de dimension 1, les pathologies de type «ensembles denses d'arcs», autorise quand même des singularités non triviales.

\section{Le théorème principal}

Théorème. Soit $\left\{v_{\varepsilon}\right\}$ une suite satisfaisant (1.1.2), (1.1.3), et de plus

$$
\begin{gathered}
\partial_{t} v_{\varepsilon}+\operatorname{div}\left(v_{\varepsilon} \otimes v_{\varepsilon}\right)=-\nabla p_{\varepsilon}+f_{\varepsilon}, \\
\operatorname{div} v_{\varepsilon}=0,
\end{gathered}
$$

avec $f_{\varepsilon} \in L_{\text {loc }}^{1}, f_{\varepsilon} \rightarrow f \in L_{\text {loc }}^{1}$ au sens des distributions.

Supposons qu'il existe dans $\mathbb{R}^{3}$ un ensemble dense $D$ de plans passant par l'axe des temps tel que, localement, supp $\sigma$ se projette sur chacun de ces plans en un ensemble de type fini.

Alors $v$ est solution de l'équation

$$
\partial_{t} v+\operatorname{div}(v \otimes v)=-\nabla p+f, \quad \operatorname{div} v=0 .
$$

Corollaire. Soit une suite $\left\{v_{\varepsilon}\right\}$ satisfaisant (1.1.2), (1.1.3) et (3.1) Si supp $\sigma$ est localement contenu dans une réunion finie de segments de courbes $C^{1}$, alors (3.2) est vrai. 
La preuve de ce théorème sera présentée à la Sect. 4. Auparavant, faisons quelques remarques:

i) Des exemples de suites stationnaires $v_{\varepsilon}$ concentrées sur des ensembles denses de dimension zéro ont été construits dans [5]. Dans ces cas, on observe que $\sigma$ est la mesure de Lebesgue, et la connaissance de $\sigma$ ne fournit bien sûr aucune information fine sur la structure des ensembles de convergence. La méthode de troncatures-fantômes de [3] permet néanmoins de conclure. Dans les cas nonstationnaires, des conditions techniques de régularité des troncatures-fantômes nous interdisent de traiter de telles pathologies, et nous obligent à faire une hypothèse forte en terme de la mesure de défaut $\sigma$.

ii) Comme il est observé dans [2], des hypothèses de régularité supplémentaires (par exemple, dans le cas stationnaire, $\nabla v_{\varepsilon}$ borné dans $L^{1}$ ) impliquent une structure raisonnable de $\sigma$ (comme somme de masses de Dirac). L'hypothèse faite ici n'est donc pas irréaliste.

iii) En passant à la limite (au sens des distributions) dans l'équation, on obtient

$$
\partial_{t} v+\operatorname{div} \mu=-\nabla q+f, \quad \operatorname{div} v=0,
$$

d'où, par différence

$$
\operatorname{div}(v \otimes v-\mu)=-\nabla(p-q) .
$$

Autrement dit, la mesure de défaut matricielle $\mu-v \otimes v$ vérifie l'équation d'Euler stationnaire.

Enfin, décrivons brièvement les idées de la preuve du théorème, pour mieux en saisir l'application éventuelle à d'autres situations:

Comme on le voit sur la formulation duale (4.3.2) des équations, deux termes bien distincts apparaissent:

$$
\int \nabla^{\perp} \partial_{t} \eta \cdot v_{\varepsilon} \text { et } \int \nabla \nabla^{\perp} \eta:\left(v_{\varepsilon} \times v_{\varepsilon}\right) .
$$

i) Le passage à la limite lorsque $\varepsilon \rightarrow 0$ s'effectue sans difficulté dans le premier terme, mais nécessite une condition de support sur la matrice $\nabla \nabla^{\perp} \eta$ du second: le choix de $\eta$ dépendant essentiellement d'une seule variable " $x$ » permet de prendre $\nabla \nabla^{\perp} \eta$ arbitraire (alors qu'à plusieurs variables, les compatibilités empêchent de résoudre; ceci explique 4.3e).

ii) Après le passage à la limite en $\varepsilon \rightarrow 0$ l'élimination de la condition de support sur $\nabla \nabla^{\perp} \eta$ (dite «méthode des troncatures fantômes») se fait automatiquement dans le second terme (grâce au choix de $\eta$ en 4.3b), mais n'est possible dans le premier que sous des conditions géométriques précises, explicitées au Lemme 4.2. En gros, ces conditions signifient que $\partial_{t}$ équivaut à $\partial_{x}$ uniformément sur les fonction de troncature introduites, c'est-à-dire que ces troncatures ont lieu sur des voisinages tubulaires d'arcs non horizontaux.

On remarque ici que l'analyse des types de (suites de) troncatures admissibles repose sur l'examen des structures respectives des termes «linéaires» et «nonlinéaires» de l'équation.

iii) La nécessité structurelle de distinguer deux types d'arcs conduit aux deux notions d'ensembles de type fini: le type $k$ et le type $k$ restreint, seuls les arcs non 
horizontaux étant autorisés dans ce dernier. La discussion de ces notions et des troncatures correspondantes est faite à la Sect. 4.1.

iv) Enfin, on remarque que c'est la régularité a priori dissymétrique (1.1.2) de la suite de solutions considérée qui rend possible l'«excision» des fragments d'arcs horizontaux: c'est le but du Lemme 4.1.1 et des Sects. 4.3c, d.

En termes d'analyse microlocale, on peut dire que seule la direction $\xi=0$ est mauvaise, inconvénient compensé par une meilleure régularité en $t$. Des idées voisines sont à l'oeuvre dans [4].

\section{Preuve du théorème}

4.1. Une discussion sur les ensembles de type fini

Introduisons d'abord la définition suivante.

Définition 4.1. $E$ de type $k$ restreint est défini comme à la Définition 2.1.1, en n'autorisant cette fois que des fonctions $\psi$ de la forme $\psi(x, t)=x-\varphi(t)$.

Le lien avec les ensembles de type fini est donné par le lemme de réduction suivant.

Lemme 4.1.1 («Lemme de réduction»). Si E est de type $k$, pour tout $\varepsilon>0$, il existe un ouvert $\omega \subset \mathbb{R}$, de mesure au plus égale à $\varepsilon$, tel que $E \backslash t^{-1}(\omega)$ soit de type $k$ restreint. Preuve. Elle s'effectue par récurrence sur $k$.

Si $k=0$, c'est évident. Supposons la propriété vraie pour un $k>0$ et soit $E$ de type $k+1$.

Soit $K_{1}, \ldots, K_{N}, \psi_{1}, \ldots, \psi_{N}$ comme dans la Définition 2.1.1, $\psi_{1} \equiv t, \psi_{j}(x, t)=x$ $-\varphi_{j}(t)(j \geqq 2)$. Choisissons, pour $j \geqq 2$, des suites décroissantes d'ouverts $\omega_{j}^{n} \supset K_{j}, \omega_{j}^{n}$ étant une réunion finie d'intervalles ouverts disjoints de longueur totale au plus $\frac{1}{n}$. Notons $\widetilde{K}_{j}=\bigcap_{n} \overline{\omega_{j}^{n}}: \widetilde{K}_{j}$ est un compact de mesure nulle, et pour tout ouvert $\omega \supset \widetilde{K}_{j}$, $\overline{\omega_{j}^{n}} \subset \omega$ à partir d'un certain rang.

Soit $\varepsilon>0$. Choisissons $\omega_{1} \supset K_{1}$, mesure $\omega_{1} \leqq \frac{\varepsilon}{2}$ : pour tout $n$,

$$
E \backslash\left(t^{-1}\left(\omega_{1}\right) \cup \bigcup_{j \geqq 2} \psi_{j}^{-1}\left(\omega_{j}^{n}\right)\right)=F_{n}
$$

est de type $k$, et il existe un ouvert $\tilde{\omega}^{n}$, mesure $\tilde{\omega}^{n} \leqq \frac{\varepsilon}{2^{n+1}}$ tel que $F_{n} \backslash t^{-1}\left(\tilde{\omega}^{n}\right)$ soit de type $k$ restreint. Posons $\tilde{\omega}=\bigcup_{n} \tilde{\omega}^{n}$; pour tous $\omega_{j} \supset \tilde{K}_{j}(j \geqq 2)$, soit $n$ tel que $\overline{\omega_{j}^{n}} \subset \omega_{j}$;
l'ensemble

$$
G=E \backslash\left(t^{-1}\left(\omega_{1} \cup \tilde{\omega}\right) \cup \bigcup_{j \geqq 2} \psi_{j}^{-1}\left(\omega_{j}\right)\right) \subset F_{n} \backslash t^{-1}(\tilde{\omega}) \subset F_{n} \backslash t^{-1}\left(\tilde{\omega}^{n}\right)
$$

est donc de type $k$ restreint, et comme mesure $\left(\omega_{1} \cup \tilde{\omega}\right) \leqq \varepsilon$, la preuve est complète.

Par ailleurs, nous aurons besoin d'utiliser des troncatures régulières de forme très spéciale, s'annulant sur un ensemble donné $E$. La construction de ces troncatures est résumée dans le lemme suivant. 
Rappelons la notation

$$
\varphi_{\varepsilon}(t)=\int \frac{1}{\varepsilon} \lambda\left(\frac{t-t^{\prime}}{\varepsilon}\right) \varphi\left(t^{\prime}\right) d t^{\prime}, \quad \int \lambda(s) d s=1, \quad 0 \leqq \lambda \leqq 1, \lambda \in C_{0}^{\infty}(\mathbb{R})
$$

pour la régularisée d'une fonction $\varphi(t)$.

Lemme 4.1.2 («Lemme des troncatures»). Soit E de type k restreint. Il existe alors $N$ suites $\left\{\chi_{n}^{1}\right\}, \ldots,\left\{\chi_{n}^{N}\right\}$ de fonctions de la forme

$$
\chi_{n}^{j}(x, t)=\tilde{\chi}_{n}^{j}\left(x-\varphi_{\varepsilon_{n}}^{j}(t)\right)
$$

(où les $\varphi^{j}$ sont Lipschitziennes à supports compacts) telles que:

i) Pout $t$ fixé, $\chi_{n}^{j}(t, \cdot) \rightarrow 1$ p.p. en $x$.

ii) Pout tous $n_{1}, \ldots, n_{N}$,

$$
E \cap \operatorname{supp}\left(\chi_{n_{1}}^{1} \ldots \chi_{n_{N}}^{N}\right) \text { est de type } k-1 \text { restreint. }
$$

Si $k=0$, (4.1.1) signifie seulement $E \cap \operatorname{supp}(\ldots)=\phi$.

Preuve. a. Soient $K_{1}, \ldots, K_{N}, \psi_{1}, \ldots, \psi_{N}\left(\psi_{i}=x-\varphi^{i}\right)$ les compacts et les fonctions correspondant à la Définition 4.1, et soient $\omega_{j}^{n}$ des suites d'ouverts choisies comme au Lemme 4.1.1.

Soit $N(n)+2$ le nombre d'intervalles de $\left(\omega_{j}^{n}\right)^{c}$, et soit $[\alpha, \beta]$ l'un deux, supposé borné: on choisira pour $\tilde{\chi}_{n}^{j}$ dans $[\alpha, \beta]$ une fonction $C^{\infty}$, comprise entre 0 et 1 , valant 1 sur l'intervalle $[\alpha+2 \eta, \beta-2 \eta]$ et à support dans $[\alpha+\eta, \beta-\eta]$; ici,

$$
\eta=\eta_{j}(n)=\frac{1}{4(N(n)+1)} \frac{1}{n}
$$

(si $4 \eta \geqq \beta-\alpha$, on prend simplement $\tilde{\chi}_{n}^{j}=0$ sur $\left.[\alpha, \beta]\right)$. On procède de même avec un intervalle non borné du type $]-\infty, \alpha]$ (par exemple): $\tilde{\chi}_{n}^{j}$ sera choisie $C^{\infty}$, valant 1 dans $] \infty, \alpha-2 \eta]$, nulle dans $[\alpha-\eta, \alpha]$.

La fonction $\tilde{\chi}_{n}^{j}$ construite vérifie donc

$$
\left\{\begin{array}{l}
\operatorname{supp} \tilde{\chi}_{n}^{j} \subset\left(\omega_{j}^{n}\right)^{c}, \tilde{\chi}_{n}^{j}=1 \text { sur } G_{n}^{j}=\text { réunion d'intervalles } \\
\text { tels que }[\alpha+2 \eta, \beta-2 \eta], \text { et mesure }\left(G_{n}^{j}\right)^{c} \leqq \frac{2}{n}
\end{array}\right.
$$

Remarquons que lorsque $n$ augmente, un intervalle de $\left(\omega_{j}^{n}\right)^{c}$ ne peut que s'agrandir, et de nouveaux intervalles de $\left(\omega_{j}^{n}\right)^{c}$ peuvent apparaître: $N(n)$ est donc croissante, $\eta(n)$ décroissante, et la suite des $G_{n}^{j}$ croît.

b. En notant $C_{0}$ un majorant des normes Lipschitz des $\varphi^{j}$, choisissons $\varepsilon_{n}>0$ telle que

$$
\left(\int \lambda|s| d s\right) C_{0} \varepsilon_{n} \leqq \inf _{j} \eta_{j}(n) .
$$

En notant $\widetilde{G}_{n}^{j}$ la réunion d'intervalles tels que $[\alpha+3 \eta, \beta-3 \eta]$, on remarque que $\widetilde{G}_{n}^{j}$ est une suite croissante, et $H_{j}=\bigcap_{n}\left(\widetilde{G}_{n}^{j}\right)^{c}$ est de mesure nulle.

Montrons alors que si $x-\varphi^{j}(t) \notin H_{j}, \chi_{n}^{j}(x, t)$ tend vers 1 lorsque $n \rightarrow+\infty$. En effet, $x-\varphi^{i}(t) \in \widetilde{G}_{n}^{j}$ pour $n$ assez grand, donc

car

$$
x-\varphi_{\varepsilon_{n}}^{j}(t)=x-\varphi^{j}(t)+\varphi^{j}(t)-\varphi_{\varepsilon_{n}}^{j}(t) \in G_{n}^{j},
$$

$$
\left|\varphi^{j}(t)-\varphi_{\varepsilon}^{j}(t)\right| \leqq \int \frac{1}{\varepsilon} \lambda\left(\frac{t-t^{\prime}}{\varepsilon}\right)\left|\varphi^{j}\left(t^{\prime}\right)-\varphi^{j}(t)\right| d t^{\prime} \leqq C_{0} \varepsilon \int \lambda|s| d s .
$$


Alors $\chi_{n}^{j}$ vaut 1 , d'après (4.1.2), ce qui prouve i).

c. Si $x-\varphi^{j}(t) \in \omega_{j}^{n}, x-\varphi_{\varepsilon_{n}}^{j}(t) \notin \operatorname{supp} \tilde{\chi}_{n}^{j}$, donc $\chi_{n}^{j}(x, t)=0$. Comme

et est donc de type $k-1$ restreint.

$$
\begin{gathered}
\operatorname{supp} \chi_{n_{1}}^{1} \ldots \chi_{n_{N}}^{N} \subset \operatorname{supp} \chi_{n_{1}}^{1} \cap \ldots \cap \operatorname{supp} \chi_{n_{N}}^{N}, \\
E \cap \operatorname{supp} \chi_{n_{1}}^{1} \ldots \chi_{n_{N}}^{N} \subset E \backslash \bigcup_{j} \psi_{j}^{-1}\left(\omega_{j}^{n_{j}}\right)
\end{gathered}
$$

4.2. Un lemme de passage à la limite

La méthode des «troncatures-fantômes» utilisée dans la preuve du théorème repose sur le lemme suivant.

Lemma 4.2. Soit $\varphi: \mathbb{R} \rightarrow \mathbb{R}$ une fonction Lipschitzienne à support compact.

Soit une suite de fonctions $\chi_{n}(x, t)$, de la forme

$$
\chi_{n}(x, t)=\tilde{\chi}_{n}\left(x-\varphi_{\varepsilon_{n}}(t)\right)
$$

(pour une suite $\tilde{\chi}_{n} \in C^{\infty}(\mathbb{R}), 0 \leqq \tilde{\chi}_{n} \leqq 1, \varepsilon_{n}>0$ ), et telle que pout $t$ fixé,

$$
\chi_{n}(x, t) \rightarrow 1 \text { p.p.en } x \text {. }
$$

Soit enfin $k \in C^{\infty}\left(\mathbb{R}^{2}\right)$, telle que pour $t$ dans un compact, $k(\cdot, t)$ est nulle pour $|x| \geqq R$.

Alors, pour tout $\tilde{\varphi} \in C_{0}^{\infty}\left(\mathbb{R}^{3}\right), v=\left(v^{1}, v^{2}\right) \in L_{\mathrm{loc}}^{1}\left(\mathbb{R}^{3}\right)$,

$$
\begin{aligned}
I_{n}= & \int_{\mathbb{R}^{3}} \nabla^{\perp} \partial_{t}\left(\tilde{\varphi}\left(x_{1}, x_{2}, t\right) \int_{-\infty}^{x_{1}} d z \int_{-\infty}^{z}\left(1-\chi_{n}(s, t)\right) k(s, t) d s\right) \\
& \times v(x, t) d x d t \rightarrow 0 \quad \text { lorsque } n \rightarrow+\infty
\end{aligned}
$$

$\left[\right.$ on a noté $\left.\nabla^{\perp} \eta\left(x_{1}, x_{2}, t\right)=\left(-\frac{\partial \eta}{\partial x_{2}}, \frac{\partial \eta}{\partial x_{1}}\right)\right]$.

Preuve: a) On a:

$$
\begin{aligned}
I_{n}= & +\int\left(-\partial_{t} \partial_{x_{2}} \tilde{\varphi} v^{1}+\partial_{t} \partial_{x_{1}} \tilde{\varphi} v^{2}\right) \\
& \times\left(\int_{-\infty}^{x_{1}} d z \int_{-\infty}^{z}\left(1-\chi_{n}(s, t)\right) k(s, t) d s\right) d x d t \\
& +\int\left(\partial_{x_{1}} \tilde{\varphi} v^{2}-\partial_{x_{2}} \tilde{\varphi} v^{1}\right)\left\{\int_{-\infty}^{x_{1}} d z \int_{-\infty}^{z} \partial_{t}(1-\chi(s, t)) k(s, t) d s\right. \\
& \left.+\int_{-\infty}^{x_{1}} d z \int_{-\infty}^{z}\left(1-\chi_{n}(s, t)\right)\left(\partial_{t} k\right)(s, t) d s\right\} d x d t \\
& +\int \partial_{t} \tilde{\varphi} v^{2}\left(\int_{-\infty}^{x_{1}}\left(1-\chi_{n}(s, t)\right) k(s, t) d s\right) d x d t \\
& +\int \tilde{\varphi} v^{2}\left\{\int_{-\infty}^{x_{1}} \partial_{t}(1-\chi(s, t)) k(s, t) d s\right. \\
& \left.+\int_{-\infty}^{x_{1}}\left(1-\chi_{n}(s, t)\right)\left(\partial_{t} k\right)(s, t) d s\right\} d x d t \\
= & (1)+(2)+(2)^{\prime}+(3)+(4)+(4)^{\prime} .
\end{aligned}
$$


b) Pour $\tilde{k}=k, \partial_{t} k$ ou $\partial_{s} k$, la suite

$$
f_{n}(z, t)=\int_{-\infty}^{z}\left(1-\chi_{n}(s, t)\right) \tilde{k}(s, t) d s
$$

converge simplement vers 0 , et, si $t$ reste dans un compact, $f_{n}(z, t)=0$ pour $z \leqq c t e$, $\left|f_{n}(z, t)\right| \leqq$ cte.

Cela implique que les fonctions

$$
\int_{-\infty}^{x_{1}} d z \int_{-\infty}^{z}\left(1-\chi_{n}(s, t)\right) \tilde{k}(s, t) d s
$$

tendent simplement vers 0 , en restant bornées sur les compacts. Donc les termes (1), $(2)^{\prime},(3)$ et $(4)^{\prime}$ tendent vers 0.

c) On a

Par conséquent

$$
\begin{aligned}
\int_{-\infty}^{z} \partial_{t}\left(1-\chi_{n}(s, t)\right) k(s, t) d s & =\int_{-\infty}^{z} \varphi_{\varepsilon_{n}}^{\prime}(t) \tilde{\chi}_{n}^{\prime}\left(s-\varphi_{\varepsilon_{n}}(t)\right) k(s, t) d s \\
& =-\varphi_{\varepsilon_{n}}^{\prime}(t) \int_{-\infty}^{z} \partial_{s}\left(1-\chi_{n}(s, t)\right) k(s, t) d s \\
& =-\varphi_{\varepsilon_{n}}^{\prime}(t)\left(1-\chi_{n}(z, t)\right) k(z, t) \\
& +\varphi_{\varepsilon_{n}}^{\prime}(t) \int_{-\infty}^{z}\left(1-\chi_{n}(s, t)\right)\left(\partial_{s} k\right)(s, t) d s
\end{aligned}
$$

$$
\begin{aligned}
(2)= & -\int\left(\partial_{x_{1}} \tilde{\varphi} v^{2}-\partial_{x_{2}} \tilde{\varphi} v^{1}\right) \varphi_{\varepsilon_{n}}^{\prime}(t)\left(\int_{-\infty}^{x_{1}}\left(1-\chi_{n}(z, t)\right) k(z, t) d z\right) d x d t \\
& +\int\left(\partial_{x_{1}} \tilde{\varphi} v^{2}-\partial_{x_{2}} \tilde{\varphi} v^{1}\right) \varphi_{\varepsilon_{n}}^{\prime}(t) \\
& \times\left(\int_{-\infty}^{x_{1}} d z \int_{-\infty}^{z}\left(1-\chi_{n}(s, t)\right)\left(\partial_{s} k\right)(s, t) d s\right) d x d t .
\end{aligned}
$$

Comme $\varphi$ est Lipschitzienne, $\left|\varphi_{\varepsilon}^{\prime}(t)\right| \leqq C_{0} \int\left|\lambda^{\prime}(\sigma)\right||\sigma| d \sigma$; le point b) implique donc $(2) \rightarrow 0$.

d) D'autre part,

$$
\begin{aligned}
(4)= & -\int \tilde{\varphi} v^{2} \varphi_{\varepsilon_{n}}^{\prime}(t)\left(1-\chi\left(x_{1}, t\right)\right) k\left(x_{1}, t\right) d x d t \\
& +\int \tilde{\varphi} v^{2} \varphi_{\varepsilon_{n}}^{\prime}(t)\left(\int_{-\infty}^{x_{1}}\left(1-\chi_{n}(s, t)\right)\left(\partial_{s} k\right)(s, t) d s\right) d x d t \\
= & (5)+(6) .
\end{aligned}
$$

Comme $\int\left|\tilde{\varphi} v^{2} k\right|\left(1-\chi_{n}\left(x_{1}, t\right)\right) d x_{1}$ est bornée et tend vers zéro pour $\left(x_{2}, t\right)$ fixé, $(5) \rightarrow 0$. Enfin, (6) $\rightarrow 0$, d'après b).

\subsection{Preuve du théorème}

a. Tout champ $w \in C_{0}^{\infty}, \operatorname{div} w=0$, s'écrit $w=\nabla^{\perp} \eta$ pour un $\eta \in C_{0}^{\infty}$. Pour montrer (3.2), il suffit de prouver

$$
\int \nabla^{\perp} \partial_{t} \eta \cdot v+\int \nabla \nabla^{\perp} \eta:(v \otimes v)=-\int \nabla^{\perp} \eta \cdot f,
$$

où: dénote la somme des produits terme à terme des matrices. 
Par l'hypothèse (3.1), on a de même, pour tout $\varepsilon>0$ et tout $\eta \in C_{0}^{\infty}$,

$$
\int \nabla^{\perp} \partial_{t} \eta \cdot v_{\varepsilon}+\int \nabla \nabla^{\perp}:\left(v_{\varepsilon} \otimes v_{\varepsilon}\right)=-\int \nabla^{\perp} \eta \cdot f_{\varepsilon} .
$$

b. Fixons $\tilde{\varphi} \in C_{0}^{\infty}\left(\mathbb{R}_{x}^{2} \times\right] 0, T[), \operatorname{supp} \tilde{\varphi} \subset\left\{x,|x| \leqq R_{0}\right\}$.

Soit $\gamma \in \mathbb{R}$, et $l \in C_{0}^{\infty}(\mathbb{R})$ : on se propose d'établir une version modifiée de (4.3.1) pour $\eta=\tilde{\varphi} l\left(x_{1}+\gamma t\right)$.

Pour ce faire, supposons que la projection $E$ de supp $\sigma \cap \operatorname{supp} \tilde{\varphi}$ sur le plan $\left(x_{1}, t\right)$ soit de type $k$.

Fixons $\varepsilon_{0}>0$ et choisissons $\varepsilon_{1}>0$ tel que

$$
\varepsilon_{1} C_{R_{0}} \sup \left|\tilde{\varphi} l^{\prime \prime}\left(x_{1}+\gamma t\right)\right| \leqq \frac{\varepsilon_{0}}{2} .
$$

D'après le lemme de réduction 4.1.1, il existe un ouvert $\omega$ de mesure au plus $\varepsilon_{1}$, tel que $F=E \backslash t^{-1}(\omega)$ soit de type $k$ restreint. Le lemme des troncatures 4.1.2 fournit alors $N_{1}$ suites de fonctions $\chi_{1, n}^{1}, \ldots, \chi_{1, n}^{N_{1}}$ (dites «première génération»). Fixons $n_{1}, \ldots, n_{N_{1}}$ : l'ensemble $F \cap \operatorname{supp}\left(\chi_{1, n_{1}}^{1} \ldots \chi_{1, n_{N_{1}}}^{n_{1}}\right)$ est de type $k-1$ restreint. En appliquant de nouveau le lemme des troncatures, on obtient $N_{2}$ suites de fonctions $\chi_{2, n}^{1}, \ldots, \chi_{2, n}^{N_{2}}$ (dites «deuxième génération»), qui dépendent bien entendu des entiers $n_{1}, \ldots, n_{N_{1}}$ choisis, telles que pour $n_{1}, \ldots, n_{N_{2}}$ arbitraires,

$$
\begin{gathered}
F \cap \operatorname{supp}\left(\chi_{1}^{1}, \ldots \chi_{1}^{N_{1}}, \chi_{2}^{1}, \ldots \chi_{2}^{N_{2}}\right) \subset F \cap \operatorname{supp}\left(\chi_{1}^{1}, \ldots \chi_{1}^{N_{1}}\right) \cap \operatorname{supp}\left(\chi_{2}^{1}, \ldots \chi_{2}^{N_{2}}\right) \\
\text { est de type } k-2 \text { restreint. }
\end{gathered}
$$

(On a omis d'écrire les indices en bas pour alléger.)

On fixe donc successivement les indices de première génération $n_{1}, \ldots, n_{N_{1}}$, puis de deuxième et ainsi de suite jusqu'à la kième génération $n_{1}, \ldots, n_{N_{k}}$ (par abus, on omet dans les $n_{j}$ l'indice de génération: l'ensemble $F \cap \operatorname{supp}\left(\chi_{1}^{1}, \ldots \chi_{1}^{N_{1}}, \chi_{2}^{1}, \ldots \chi_{k}^{N_{k}}\right.$, est alors de type 0 restreint. On prend finalement $n_{1}, \ldots, n_{N_{k+1}}$ arbitraires, et l'on écrit l'équation (4.3.2) pour le potential $\eta=\tilde{\varphi} \widetilde{l}\left(x_{1}, t\right)$, où

$$
\widetilde{l}\left(x_{1}, t\right)=\int_{-\infty}^{x_{1}} d z \int_{-\infty}^{z} \chi(s, t) l^{\prime \prime}(s+\gamma t) d s
$$

$\chi$ désignant le produit des troncature $\chi_{1, n_{1}}^{1} \ldots \chi_{k+1, n_{N_{k}+1}}^{N_{k+1}}$.

c. On a en fait

et

$$
\begin{aligned}
\int \nabla \nabla^{\perp} \eta:\left(v_{\varepsilon} \otimes v_{\varepsilon}\right)= & \int \tilde{\varphi} \tilde{l}^{\prime \prime} v_{\varepsilon}^{1} v_{\varepsilon}^{2}+\int\left(\nabla \tilde{\varphi}^{t} \nabla^{\perp} \tilde{l}\right):\left(v_{\varepsilon} \otimes v_{\varepsilon}\right) \\
& +\int\left(\nabla \tilde{l}^{t} \nabla^{\perp} \tilde{\varphi}\right):\left(v_{\varepsilon} \otimes v_{\varepsilon}\right)+\int \nabla \nabla^{\perp} \tilde{\varphi}:\left(v_{\varepsilon} \otimes v_{\varepsilon}\right) \tilde{l} \\
= & (1)+(2)+(3)+(4),
\end{aligned}
$$

$$
\tilde{l}^{\prime \prime}\left(x_{1}, t\right)=\chi\left(x_{1}, t\right) l^{\prime \prime}\left(x_{1}+\gamma t\right)
$$

(' signifie une dérivée en $x_{1}$ ).

On scinde (1) en

$$
(1)=\int_{\omega} d t \int_{\mathbb{R}^{2}} \tilde{\varphi} \chi l^{\prime \prime} v_{\varepsilon}^{1} v_{\varepsilon}^{2} d x+\int_{\omega^{c}} d t \int_{\mathbb{R}^{2}} \tilde{\varphi} \chi l^{\prime \prime} v_{\varepsilon}^{1} v_{\varepsilon}^{2} d x=(1)^{\prime}+(1)^{\prime \prime},
$$

et $\left|(1)^{\prime}\right| \leqq \frac{\varepsilon_{0}}{2}$ par le choix de $\omega$ et $(4.3 .3)$. 
On passe maintenant à la limite lorsque $\varepsilon \rightarrow 0$ dans les termes (1)", (2), (3) et (4). Par construction, l'intégrale dans (1)" a lieu sur un compact disjoint de supp $\sigma$ : sur un tel compact, la convergence de $v_{\varepsilon}$ vers $v$ est forte, donc

$$
(1)^{\prime \prime} \rightarrow \int_{\omega^{c}} d t \int_{\mathbb{R}^{2}} \tilde{\varphi} \chi l^{\prime \prime} v^{1} v^{2} d x .
$$

Les termes (2), (3), (4) en revanche tendent vers des termes (2)', (3)', (4)' de même forme, où $v_{\varepsilon} \otimes v_{\varepsilon}$ a été remplacé par $\mu$.

Finalement,

$$
\int \nabla^{\perp} \partial_{t} \eta \cdot v_{\varepsilon} \rightarrow \int \nabla^{\perp} \partial_{t} \eta \cdot v
$$

et

$$
\int \nabla^{\perp} \eta \cdot f_{\varepsilon} \rightarrow \int \nabla^{\perp} \eta \cdot f
$$

d. A l'issue de c, on a donc obtenu

$$
\left|\int \nabla^{\perp} \eta_{t} \cdot v+\int_{\omega^{c}} d t \int \tilde{\varphi} \chi l^{\prime \prime} v^{1} v^{2} d x+(2)^{\prime}+(3)^{\prime}+(4)^{\prime}+\int \nabla^{\perp} \eta \cdot f\right| \leqq \frac{\varepsilon_{0}}{2},
$$

d'où

$$
\left|\int \nabla^{\perp} \eta_{t} \cdot v+\int \tilde{\varphi} \chi l^{\prime \prime} v^{1} v^{2}+(2)^{\prime}+(3)^{\prime}+(4)^{\prime}+\int \nabla^{\perp} \eta \cdot f\right| \leqq \varepsilon_{0} .
$$

On va maintenant faire disparaître une à une, génération par génération, les troncatures qui forment $\chi$, en appliquant de façon répétée le Lemme 4.2 (c'est pourquoi la méthode est dite «des troncatures-fantômes»).

On fait tendre d'abord $n_{N_{k+1}}$ vers l'infini, toutes les précédentes troncatures restant fixées: le Lemme 4.2, appliqué avec $k(s, t)=$ le produit des précédentes troncatures $\times l^{\prime \prime}(s+\gamma t)$, montre qu'on peut supprimer la dernière troncature $\tilde{\chi}$ dans le terme $\int \nabla^{\perp} \partial_{t} \eta \cdot v$.

Dans le terme $\int \tilde{\varphi} \chi l^{\prime \prime} v^{1} v^{2}$, la suppression de $\tilde{\chi}$ résulte du théorème de convergence dominée. Dans les termes $(2)^{\prime},(3)^{\prime},(4)^{\prime}$ et $\int \nabla^{\perp} \eta \cdot f$, le passage à la limite résulte de ce que

$$
\begin{aligned}
\tau & \rightarrow \int_{-\infty}^{x_{1}} d z \int_{-\infty}^{z} k(s, t) d s, \\
\tau^{\prime} & \rightarrow \int_{-\infty}^{x_{1}} k(s, t) d s
\end{aligned}
$$

uniformément sur tout compact;

en effet, pour $\left(x_{1}, t\right)$ dans un compact,

$$
\left|\int_{-\infty}^{x_{1}}(1-\tilde{\chi}) k d s\right| \leqq|k|_{L^{\infty}} \sup _{t} \int_{-\infty}^{+\infty}(1-\tilde{\chi}) d s \leqq|k|_{L^{\infty}} \frac{2}{n_{N_{k+1}}}
$$

et

$$
\left|\int_{-\infty}^{x_{1}} d z \int_{-\infty}^{z}(1-\tilde{\chi}) k d s\right| \leqq \mathrm{cte}|k|_{L^{\infty}} \frac{2}{n_{N_{k+1}}}
$$

par construction des troncatures (voir Lemme 4.1.2).

On obtient ainsi (4.3.4) avec une nouvelle fonction $\eta$ pour laquelle $\chi$ contient une troncature de moins (la dernière). 
En faisant disparaître successivement toutes les troncatures comme plus haut, on obtient finalement $|I| \leqq \varepsilon_{0}$, où

$$
\begin{aligned}
I= & \int\left(\nabla^{\perp} \partial_{t} \tilde{\varphi} l\right) \cdot v+\int \tilde{\varphi} \nabla \nabla^{\perp} l:(v \otimes v)+\int \nabla \tilde{\varphi}^{t} \nabla^{\perp} l: \mu \\
& +\int \nabla l^{t} \nabla^{\perp} \tilde{\varphi}: \mu+\int \nabla \nabla^{\perp} \tilde{\varphi}: \mu l+\int \nabla^{\perp} \tilde{\varphi} l \cdot f,
\end{aligned}
$$

avec $l=l\left(x_{1}, t\right)$.

Comme $\varepsilon_{0}$ est arbitraire, on a fait

$$
I=0 .
$$

e. Soit $\pi$ un plan de $D$ : on peut toujours choisir des coordonnées en sorte que $\pi$ soit le plan $\left(x_{1}, t\right)$, et l'hypothèse du théorème nous place alors dans la situation de $b$. On obtient donc (4.3.6) avec $l=l(d \cdot x+\gamma t)\left(d=(\pi \cap t=0) \cap S^{1}, \gamma \in \mathbb{R}\right)$, où $l$ est à support compact.

Il est facile de voir, à l'aide de la transformation de Radon, que des combinaisons linéaires finies de fonctions telles que $l(d \cdot x+\gamma t)$ approchent une fonction test donnée $\eta$ en norme $C^{2}$ : en passant à la limite dans (4.3.6), on trouve donc

$$
\begin{aligned}
& \int \nabla^{\perp} \partial_{t} \tilde{\varphi} \eta \cdot v+\int \tilde{\varphi} \nabla \nabla^{\perp} \eta: v \otimes v+\int \nabla \tilde{\varphi}^{t} \nabla^{\perp} \eta: \mu \\
& \quad+\int \nabla \eta^{t} \nabla^{\perp} \tilde{\varphi}: \mu+\int \nabla \nabla^{\perp} \tilde{\varphi}: \mu \eta+\int \nabla^{\perp} \tilde{\varphi} \eta \cdot f=0 .
\end{aligned}
$$

Si l'on a pris soin de choisir $\tilde{\varphi}=1$ prés de supp $\eta$, on trouve (4.3.1), ce qui complète la preuve.

\section{Bibliographie}

1. Di Perna, R., Majda, A.: Oscillations and concentrations in weak solutions of the incompressible fluid equations. Commun. Math. Phys. 108, 667-689 (1987)

2. Di Perna, R., Majda, A.: Concentrations in regularizations for 2-D incompressible flow. Commun. Pure Appl. Math. 40, 301-345 (1987)

3. Di Perna, R., Majda, A.: Reduced Hausdorff dimension and concentration-cancellation for 2-D incompressible flow. J. Am. Math. Soc. 1, 59-95 (1988)

4. Gerard, P.: Compacité par compensation et régularité 2-microlocale, Séminaire d'EDP (1988-1989), Ecole Polytechnique, Paris, et Article à paraître

5. Greengard, C., Thomann, E.: On Di Perna-Majda Concentration sets for two-dimensional incompressible flow. Commun. Pure Appl. Math. 41, 295-303 (1988)

6. Lions, P.L.: The concentration-compactness principle in the calculus of variations: The limit case, Part I and Part II. Rev. Mat. Iberoamericana, Vol. 1 et 2, 145-201 (1985)

Communicated by A. Jaffe

Received February 14, 1989; in revised form September 25, 1989 